\title{
The Dirichlet problem for elliptic equation with several singular coefficients
}

\author{
Tuhtasin G. Ergashev \\ Communicated by Ravshan Ashurov
}

\begin{abstract}
Recently found all the fundamental solutions of a multidimensional singular elliptic equation are expressed in terms of the well-known Lauricella hypergeometric function in many variables. In this paper, we find a unique solution of the Dirichlet problem for an elliptic equation with several singular coefficients in explicit form. When finding a solution, we use decomposition formulas and some adjacent relations for the Lauricella hypergeometric function in many variables.
\end{abstract}

Keywords. Dirichlet problem, multidimensional elliptic equations, singular coefficients, decomposition formulas, Lauricella hypergeometric function.

2010 Mathematics Subject Classification. 35A08, 35J25, 35J70, 35 J75.

\section{Introduction}

It is known that the theory of boundary value problems for degenerate equations and equations with singular coefficients is one of the rapidly developing parts of the modern theory of partial differential equations, which is encountered in solving many important questions of an applied nature, for example, [3, 11]. A detailed bibliography and summary of studies of the basic boundary-value equations for degenerate equations of various types, in particular, for elliptic equations with singular coefficients, can be found in monographs [4, 14, 33, 34]. In addition, generalized axisymmetric potentials have been studied using various methods $[2,9,10,12,13,19,22,24,35,36]$. Omitting a huge bibliography in which various local and non-local boundary-value problems for mixed-type equations containing elliptic equations with singular coefficients are studied, we note some papers which are close to the present work. In the work [15], fundamental solutions were constructed for the bi-axially symmetric Helmholtz equation, and in [29-31] the explicit solutions of the Dirichlet and Dirichlet-Neumann problems in one quarter of a circle was found.

Dirichlet and Dirichlet-Neumann problems for elliptic equation with one singular coefficient in some part of ball were investigated by Agostinelli [1] and 
Olevskii [27]. Recently, Nazipov published a paper devoted to the investigation of the Tricomi problem in a mixed domain consisting of hemisphere and cone [25]. Fundamental solutions for the following three-dimensional elliptic equations with two and three singular coefficients

$$
u_{x x}+u_{y y}+u_{z z}+\frac{2 \alpha}{x} u_{x}+\frac{2 \beta}{y} u_{y}=0,0<2 \alpha, 2 \beta<1
$$

and

$$
u_{x x}+u_{y y}+u_{z z}+\frac{2 \alpha}{x} u_{x}+\frac{2 \beta}{y} u_{y}+\frac{2 \gamma}{z} u_{z}=0,0<2 \alpha, 2 \beta, 2 \gamma<1
$$

were constructed, respectively, in [21] and [16]. For equations (1) and (2), the Dirichlet, Neumann and Holmgren problems [20,26,32] were solved in some parts of the ball.

In this paper, we study the Dirichlet problem for the equation

$$
H_{\alpha}^{(m, n)}(u) \equiv \sum_{i=1}^{m} u_{x_{i} x_{i}}+\sum_{k=1}^{n} \frac{2 \alpha_{k}}{x_{k}} u_{x_{k}}=0,
$$

where $m \geq 2,0<n \leq m ; \alpha=\left(\alpha_{1}, \cdots, \alpha\right)$ and $\alpha_{k}$ are constants with $0<$ $2 \alpha_{k}<1$. Hereinafter in the present work, unless there are other reservations, the natural number $k$ will vary from 1 to $n$, inclusive.

\section{Preliminaries}

Below we give some formulas for Euler gamma-function, Gauss hypergeometric function, multiple Lauricella hypergeometric function (that is, Lauricella hypergeometric function in several variables), which will be used in the next sections.

It is known that the Euler gamma-function $\Gamma(a)$ has properties [7, pp. 17-19, (2), (10), (15)]

$$
\Gamma(a+m)=\Gamma(a)(a)_{m} ; \quad \Gamma\left(a+\frac{1}{2}\right)=\frac{\sqrt{\pi} \Gamma(2 a)}{2^{2 a-1} \Gamma(a)}, \quad \Gamma\left(\frac{1}{2}\right)=\sqrt{\pi} .
$$

Here $(a)_{m}$ is a Pochhammer symbol, for which an equality

$$
(a)_{m+n}=(a)_{m}(a+m)_{n}
$$

is true $[7, \mathrm{p} .67,(5)]$. 
A function

$$
F(a, b ; c ; x) \equiv F\left[\begin{array}{c}
a, b ; \\
c ;
\end{array}\right]:=\sum_{m=0}^{\infty} \frac{(a)_{m}(b)_{m}}{(c)_{m} m !} x^{m}, c \neq 0,-1,-2, \cdots ;|x|<1
$$

is known as the Gauss hypergeometric function and an equality

$$
F(a, b ; c ; 1)=\frac{\Gamma(c) \Gamma(c-a-b)}{\Gamma(c-a) \Gamma(c-b)} \quad[c \neq 0,-1, \cdots ; \operatorname{Re}(c-a-b)>0]
$$

holds $[7$, c.73, (14)]. Moreover, the following autotransformer formula $[7$, p.76, (22)]

$$
F(a, b ; c ; x)=(1-x)^{-b} F\left(c-a, b ; c ; \frac{x}{x-1}\right)
$$

is valid.

The Lauricella hypergeometric function in $n$ variables has a form [23]

$$
\begin{gathered}
F_{A}^{(n)}\left(a, b_{1}, \cdots, b_{n} ; c_{1}, \cdots, c_{n} ; x_{1}, \cdots, x_{n}\right) \equiv F_{A}^{(n)}\left[\begin{array}{c}
a, b_{1}, \cdots, b_{n} ; \\
c_{1}, \cdots, c_{n} ;
\end{array} x_{1}, \cdots, x_{n}\right] \\
=\sum_{m_{1}, \cdots m_{n}=0}^{\infty} \frac{(a)_{m_{1}+\cdots+m_{n}}\left(b_{1}\right)_{m_{1}} \cdots\left(b_{n}\right)_{m_{n}}}{\left(c_{1}\right)_{m_{1}} \cdots\left(c_{n}\right)_{m_{n}}} \frac{x_{1}^{m_{1}}}{m_{1} !} \cdots \frac{x_{n}^{m_{n}}}{m_{n} !}, \\
{\left[c_{k} \neq 0,-1,-2, \cdots ; k=\overline{1, n} ;\left|x_{1}\right|+\cdots+\left|x_{n}\right|<1\right] .}
\end{gathered}
$$

For a given multiple hypergeometric function, it is useful to fund a decomposition formula which would express the multivariable hypergeometric function in terms of products of several simpler hypergeometric functions involving fewer variables. Burchnall and Chaundy $[5,6]$ systematically presented a number of expansion and decomposition formulas for some double hypergeometric functions in series of simpler hypergeometric functions. For example, the Appell function

$$
\begin{gathered}
F_{2}\left(a, b_{1}, b_{2} ; c_{1}, c_{2} ; x, y\right)=\sum_{m, n=0}^{\infty} \frac{(a)_{m+n}\left(b_{1}\right)_{m}\left(b_{2}\right)_{n}}{\left(c_{1}\right)_{m}\left(c_{2}\right)_{n}} \frac{x^{m}}{m !} \frac{y^{n}}{n !}, \\
{\left[c_{1}, c_{2} \neq 0,-1,-2, \cdots ;|x|+|y|<1\right]}
\end{gathered}
$$

has the expansion [5]

$$
F_{2}\left(a, b_{1}, b_{2} ; c_{1}, c_{2} ; x, y\right)
$$

$$
=\sum_{i=0}^{\infty} \frac{(a)_{i}\left(b_{1}\right)_{i}\left(b_{2}\right)_{i}}{i !\left(c_{1}\right)_{i}\left(c_{2}\right)_{i}} x^{i} y^{i} F\left(a+i, b_{1}+i ; c_{1}+i ; x\right) F\left(a+i, b_{2}+i ; c_{2}+i ; y\right) \text {. }
$$


The Burchnall-Chaundy method, which is limited to functions of two variables, is based on the following mutually inverse symbolic operators [5]

$$
\nabla(h)=\frac{\Gamma(h) \Gamma\left(\delta_{1}+\delta_{2}+h\right)}{\Gamma\left(\delta_{1}+h\right) \Gamma\left(\delta_{2}+h\right)}, \quad \Delta(h)=\frac{\Gamma\left(\delta_{1}+h\right) \Gamma\left(\delta_{2}+h\right)}{\Gamma(h) \Gamma\left(\delta_{1}+\delta_{2}+h\right)},
$$

where $\delta_{1}=x \frac{\partial}{\partial x}$ and $\delta_{2}=y \frac{\partial}{\partial y}$.

In order to generalize the operators $\nabla(h)$ and $\Delta(h)$, defined in (9), A. Hasanov and H. M. Srivastava $[17,18]$ introduced the operators

$$
\begin{aligned}
& \tilde{\nabla}_{z_{1} ; z_{2}, \cdots, z_{n}}(h)=\frac{\Gamma(h) \Gamma\left(\delta_{1}+\cdots+\delta_{n}+h\right)}{\Gamma\left(\delta_{1}+h\right) \Gamma\left(\delta_{2}+\cdots+\delta_{n}+h\right)}, \\
& \tilde{\Delta}_{z_{1} ; z_{2}, \cdots, z_{n}}(h)=\frac{\Gamma\left(\delta_{1}+h\right) \Gamma\left(\delta_{2}+\cdots+\delta_{n}+h\right)}{\Gamma(h) \Gamma\left(\delta_{1}+\cdots+\delta_{n}+h\right)},
\end{aligned}
$$

where $\delta_{k}=z_{k} \frac{\partial}{\partial z_{k}}$, with the help of which they managed to find decomposition formulas for a whole class of hypergeometric functions in several variables. For example, the hypergeometric Lauricella function $F_{A}^{(n)}$, defined by formula (8) has the decomposition formula [17]

$$
\begin{gathered}
F_{A}^{(n)}\left(a, b_{1}, \cdots, b_{n} ; c_{1}, \cdots, c_{n} ; z_{1}, \cdots, z_{n}\right) \\
=\sum_{m_{2}, \cdots, m_{n}=0}^{\infty} \frac{(a)_{m_{2}+\cdots+m_{n}}\left(b_{1}\right)_{m_{2}+\cdots+m_{n}}\left(b_{2}\right)_{m_{2}} \cdots\left(b_{n}\right)_{m_{n}}}{m_{2} ! \cdots m_{n} !\left(c_{1}\right)_{m_{2}+\cdots+m_{n}}\left(c_{2}\right)_{m_{2}} \cdots\left(c_{n}\right)_{m_{n}}} z_{1}^{m_{2}+\cdots+m_{n}} z_{2}^{m_{2}} \cdots z_{n}^{m_{n}} \\
\times F\left(a+m_{2}+\cdots+m_{n}, b_{1}+m_{2}+\cdots+m_{n} ; c_{1}+m_{2}+\cdots+m_{n} ; z_{1}\right) \\
\times F_{A}^{(n-1)}\left(a+m_{2}+\cdots+m_{n}, b_{2}+m_{2}, \cdots, b_{n}+m_{n} ;\right. \\
\left.c_{2}+m_{2}, \cdots, c_{n}+m_{n} ; z_{2}, \cdots, z_{n}\right), n \in \mathrm{N} \backslash\{1\}
\end{gathered}
$$

However, due to the recurrence of formula (12), additional difficulties may arise in the applications of this expansion. Further study of the properties of operators (10) and (11) showed that formula (12) can be reduced to a more convenient form.

Lemma 2.1. [8] The following decomposition formula holds true at $n \in \mathrm{N} \backslash\{1\}$

$$
\begin{aligned}
& F_{A}^{(n)}\left(a, b_{1}, b_{2}, \cdots, b_{n} ; c_{1}, c_{2}, \cdots, c_{n} ; z_{1}, \cdots, z_{n}\right) \\
= & \sum_{\substack{m_{i, j}=0 \\
(2 \leq i \leq j \leq n)}}^{\infty} \frac{(a)_{A(n, n)}}{m_{i j} !} \prod_{k=1}^{n}\left[\frac{\left(b_{k}\right)_{B(k, n)}}{\left(c_{k}\right)_{B(k, n)}} z_{k}^{B(k, n)} F(a+A(k, n),\right.
\end{aligned}
$$




$$
\left.\left.b_{k}+B(k, n) ; c_{k}+B(k, n) ; z_{k}\right)\right]
$$

where

$$
A(k, n)=\sum_{i=2}^{k+1} \sum_{j=i}^{n} m_{i, j}, B(k, n)=\sum_{i=2}^{k} m_{i, k}+\sum_{i=k+1}^{n} m_{k+1, i} .
$$

The formula (13) is proved by the method mathematical induction [8].

It should be noted here that the sum $\sum_{k=1}^{n} B(k, n)$ has the parity property, which plays an important role in the calculation of the some values of hypergeometric functions. In fact, by virtue of equality

$$
\sum_{k=2}^{n} \sum_{i=2}^{k} m_{i, k}=\sum_{k=1}^{n-1} \sum_{i=k+1}^{n} m_{k+1, i}
$$

we obtain

$$
\sum_{k=1}^{n} B(k, n)=2 \sum_{k=2}^{n} \sum_{i=2}^{k} m_{i, k}=2 \sum_{k=1}^{n-1} \sum_{i=k+1}^{n} m_{k+1, i} .
$$

In the present paper, $\mathrm{R}_{m}^{n+}$ denotes $1 / 2^{n}$ part of the Euclidean space $\mathrm{R}^{m}$ :

$$
\mathbf{R}_{m}^{n+}:=\left\{\left(x_{1}, \cdots, x_{m}\right): x_{1}>0, \cdots, x_{n}>0,1 \leq n \leq m, m \geq 2\right\} .
$$

All the fundamental solutions of equation (3) in the domain $\mathrm{R}_{m}^{n+}$ were found in [8], and we will use one of these solutions in the study of the problem:

$$
\begin{gathered}
q_{n}(\mathrm{x} ; \xi)=\gamma_{n} \prod_{i=1}^{n}\left[\left(x_{i} \xi_{i}\right)^{1-2 \alpha_{i}}\right] \\
\times r^{-2 \bar{\alpha}_{n}} F_{A}^{(n)}\left(\bar{\alpha}_{n}, 1-\alpha_{1}, \cdots, 1-\alpha_{n} ; 2-2 \alpha_{1}, \cdots, 2-2 \alpha_{n} ; \sigma\right),
\end{gathered}
$$

where

$$
\begin{gathered}
\mathrm{x}:=\left(x_{1}, \cdots, x_{m}\right), \xi:=\left(\xi_{1}, \cdots, \xi_{m}\right), \sigma:=\left(\sigma_{1}, \cdots, \sigma_{n}\right) ; \\
0<2 \alpha_{1}, \cdots, 2 \alpha_{n}<1 ; \\
\bar{\alpha}_{n}=\frac{m-2}{2}+n-\alpha_{1}-\cdots-\alpha_{n} ; \\
\gamma_{n}=2^{2 \bar{\alpha}_{n}-m} \frac{\Gamma\left(\bar{\alpha}_{n}\right)}{\pi^{m / 2}} \prod_{j=1}^{n} \frac{\Gamma\left(1-\alpha_{j}\right)}{\Gamma\left(2-2 \alpha_{j}\right)}
\end{gathered}
$$




$$
r^{2}=\sum_{i=1}^{m}\left(x_{i}-\xi_{i}\right)^{2}, r_{k}^{2}=\left(x_{k}+\xi_{k}\right)^{2}+\sum_{i=1, i \neq k}^{m}\left(x_{i}-\xi_{i}\right)^{2}, \sigma_{k}=1-\frac{r_{k}^{2}}{r^{2}} .
$$

It is easy to verify that the fundamental solution $q_{n}(\mathrm{x} ; \xi)$ has the property

$$
\left.q_{n}(\mathrm{x} ; \xi)\right|_{x_{k}=0}=0 .
$$

\section{Formulation of the problem and the uniqueness of the solution}

Let $\Omega \subset \mathrm{R}_{m}^{n+}$ be a finite simple-connected domain bounded by planes $x_{1}=0$, $\cdots, x_{n}=0$ and by smooth $m$-dimensional surface $S$. The intersection of this surface with plane $x_{k}=0$ is denoted by $\chi_{k}$. Designate as the domain $S_{k}$, a hyperplane $O x_{1} \cdots x_{k-1} x_{k+1} \cdots x_{m}$, bounded by $x_{k}=0\left(0<x_{l}<a_{l},-b_{s}<\right.$ $\left.x_{s}<c_{s}, l=\overline{1, n}, l \neq k ; n<s \leq m\right)$ and by a curve $\chi_{k}$. Here $a_{l}, b_{s}$ and $c_{s}$ are positive constants. We introduce the notation:

$$
\tilde{x}_{k}:=\left(x_{1}, \cdots, x_{k-1}, x_{k+1}, \cdots, x_{n}, \cdots, x_{m}\right) \in S_{k} \subset \mathbf{R}_{m-1}^{(n-1)+} \subset \mathbf{R}^{m-1} .
$$

Dirichlet problem. To find a function $u(\mathrm{x}) \in C(\bar{\Omega}) \cap C^{2}(\Omega)$, satisfying equation (3) in $\Omega$ and conditions

$$
\begin{aligned}
\left.u\right|_{x_{k}=0} & =\tau_{k}\left(\tilde{x}_{k}\right), \quad \tilde{x}_{k} \in \bar{S}_{k}, \\
\left.u\right|_{S} & =\varphi(\mathrm{x}), \quad \mathrm{x} \in \bar{S},
\end{aligned}
$$

where $\tau_{k}\left(\tilde{x}_{k}\right)$ and $\varphi(\mathrm{x})$ are given continuous functions fulfilling the following matching conditions:

$$
\begin{gathered}
\tau_{i}\left(0, \cdots, 0, x_{n+1}, \cdots, x_{m}\right)=\tau_{j}\left(0, \cdots, 0, x_{n+1}, \cdots, x_{m}\right), i \neq j, i, j=\overline{1, n} \\
\left.\tau_{k}\left(\tilde{x}_{k}\right)\right|_{\chi_{k}}=\left.\varphi(\mathrm{x})\right|_{\chi_{k}} .
\end{gathered}
$$

One can readily check the validity of the following relation

$$
\begin{aligned}
& x_{1}^{2 \alpha_{1}} \cdots x_{n}^{2 \alpha_{n}}\left[u H_{\alpha}^{(m, n)}(w)-w H_{\alpha}^{(m, n)}(u)\right] \\
= & \sum_{i=1}^{m} \frac{\partial}{\partial x_{i}}\left[x_{1}^{2 \alpha_{1}} \cdots x_{n}^{2 \alpha_{n}}\left(u w_{x_{i}}-w u_{x_{i}}\right)\right], n \leq m .
\end{aligned}
$$

Let $\Omega_{\varepsilon}$ be a sub-domain of $\Omega$ at a distance $\varepsilon>0$ from its boundary $\partial \Omega=$ $\bigcup_{i=1}^{n} S_{i} \cup S$ and

$$
\frac{\partial}{\partial \mathbf{n}}=\sum_{i=1}^{m} \cos \left(\mathbf{n}, x_{i}\right) \cdot \frac{\partial}{\partial x_{i}}
$$


$\mathbf{n}$ is outer normal to $\partial \Omega$.

Integrate both sides of above given equality on the domain $\Omega_{\varepsilon}$ and use the classical formula of Gauss-Ostrogadsky:

$$
\begin{gathered}
\int_{\Omega_{\varepsilon}} x_{1}^{2 \alpha_{1}} \cdots x_{n}^{2 \alpha_{n}}\left[u H_{\alpha}^{(m, n)}(w)-w H_{\alpha}^{(m, n)}(u)\right] d x_{1} \cdots d x_{m} \\
=\int_{\partial \Omega_{\varepsilon}} x_{1}^{2 \alpha_{1}} \cdots x_{n}^{2 \alpha_{n}} \sum_{i=1}^{m}\left(u w_{x_{i}}-w u_{x_{i}}\right) \cos \left(\mathbf{n}, x_{i}\right) d \vartheta .
\end{gathered}
$$

Using the equality

$$
x_{1}^{2 \alpha_{1}} \cdots x_{n}^{2 \alpha_{n}}\left[u H_{\alpha}^{(m, n)}(u)+\sum_{i=1}^{m}\left(\frac{\partial u}{\partial x_{i}}\right)^{2}\right]=\sum_{i=1}^{m} \frac{\partial}{\partial x_{i}}\left(x_{1}^{2 \alpha_{1}} \cdots x_{n}^{2 \alpha_{n}} u \frac{\partial u}{\partial x_{i}}\right),
$$

we obtain

$$
\begin{gathered}
\int_{\Omega_{\varepsilon}} x_{1}^{2 \alpha_{1}} \cdots x_{n}^{2 \alpha_{n}} u H_{\alpha}^{(m, n)}(u) d x_{1} \cdots d x_{m} \\
+\int_{\Omega_{\varepsilon}} x_{1}^{2 \alpha_{1}} \cdots x_{n}^{2 \alpha_{n}} \sum_{i=1}^{m}\left(\frac{\partial u}{\partial x_{i}}\right)^{2} d x_{1} \cdots d x_{m} \\
=\int_{\Omega_{\varepsilon}} \sum_{i=1}^{m} \frac{\partial}{\partial x_{i}}\left(x_{1}^{2 \alpha_{1}} \cdots x_{n}^{2 \alpha_{n}} u \frac{\partial u}{\partial x_{i}}\right) d x_{1} \cdots d x_{m} .
\end{gathered}
$$

Applying again the formula of Gauss-Ostrogradsky to this equality and letting $\varepsilon \rightarrow 0$, we get

$$
\begin{gathered}
\int_{\Omega_{\varepsilon}} x_{1}^{2 \alpha_{1}} \cdots x_{n}^{2 \alpha_{n}} \sum_{i=1}^{m}\left(\frac{\partial u}{\partial x_{i}}\right)^{2} d x_{1} \cdots d x_{m} \\
=\sum_{k=1}^{n} \int_{S_{k}} x_{1}^{2 \alpha_{1}} \cdots x_{k-1}^{2 \alpha_{k-1}} x_{k+1}^{2 \alpha_{k+1}} \cdots x_{n}^{2 \alpha_{n}} \tau_{k} \nu_{k} d S_{k} \\
+\int_{S} x_{1}^{2 \alpha_{1}} \cdots x_{n}^{2 \alpha_{n}} \varphi \frac{\partial u}{\partial \mathbf{n}} d S
\end{gathered}
$$

where

$$
\nu_{k}\left(\tilde{x}_{k}\right):=\left.\left(x_{k}^{2 \alpha_{k}} \frac{\partial u}{\partial x_{k}}\right)\right|_{x_{k}=0} .
$$

To prove the uniqueness of the solution, as usual, we suppose that the problem has two $v, w$ solutions. Denoting $u=v-w$ we have that satisfies homogeneous 
Dirichlet problem $\left(\tau_{k}=0, \quad \varphi=0\right)$. Further we have to prove that the homogeneous problem has only trivial solution. In this case from (23) one can easily get

$$
\int_{\Omega_{\varepsilon}} x_{1}^{2 \alpha_{1}} \cdots x_{n}^{2 \alpha_{n}} \sum_{i=1}^{m}\left(\frac{\partial u}{\partial x_{i}}\right)^{2} d x_{1} \cdots d x_{m}=0 .
$$

Hence, it follows that $u_{x_{1}}=\cdots=u_{x_{m}}=0$, which implies that $u$ is a constant function. Considering homogeneous conditions (19) and (20), we conclude that $u(\mathrm{x}) \equiv 0$ in $\bar{\Omega}$.

\section{The existence of the solution}

We prove the existence of the solution in a special case of the domain $\Omega$ in order to get the solution in an explicit form. Assume $R=a_{k}=b_{k}=c_{k}$ and let

$$
\begin{aligned}
\Omega=\{ & \mathrm{x}: x_{1}^{2}+\cdots+x_{m}^{2}<R^{2}, x_{1}>0, \cdots, x_{n}>0, \\
& \left.-R<x_{n+1}<R, \cdots,-R<x_{m}<R\right\} .
\end{aligned}
$$

We find a solution of considered problem using method Green's functions [28]. Therefore, first we give a definition of Green's function for the formulated problem.

Definition 4.1. We call the function $G(\mathrm{x} ; \xi)$ as Green's function of the Dirichlet problem, if it satisfies the following conditions:

(i) this function is a regular solution of equation (3) in the domain $\Omega$, expect at the point $\xi$, which is any fixed point of $\Omega$;

(ii) it satisfies boundary conditions

$$
\left.G(\mathrm{x} ; \xi)\right|_{x_{k}=0}=0,\left.\quad G(\mathrm{x} ; \xi)\right|_{S}=0 ;
$$

(iii) it can be represented as

$$
G(\mathrm{x} ; \xi)=q_{n}(\mathrm{x} ; \xi)+q_{n}^{*}(\mathrm{x} ; \xi),
$$

where $q_{n}(\mathrm{x} ; \xi)$ is the fundamental solution found earlier (see formula (16))

(iv) function

$$
q_{n}^{*}(\mathrm{x} ; \xi)=-\left(\frac{a}{R_{0}}\right)^{2 \bar{\alpha}_{n}} q_{n}(\mathrm{x} ; \bar{\xi})
$$

is a regular solution of equation (3) in the domain $\Omega$. Here

$$
\bar{\xi}:=\left(\bar{\xi}_{1}, \cdots, \bar{\xi}_{m}\right), \bar{\xi}_{i}=\frac{a^{2}}{R_{0}^{2}} \xi_{i}, R_{0}^{2}=\xi_{1}^{2}+\cdots+\xi_{m}^{2} .
$$


Excise a small ball with its center at $\xi$ and with radius $\rho>0$ from the domain $\Omega$. Designate the sphere of the excised ball as $C_{\rho}$ and by $\Omega_{\rho}$ denote the remaining part of $\Omega$.

Applying formula (22), we obtain

$$
\begin{gathered}
\int_{C_{\rho}} \mathbf{x}^{(2 \alpha)}\left[u(\mathrm{x}) \frac{\partial G(\mathrm{x} ; \xi)}{\partial \mathbf{n}}-G(\mathrm{x} ; \xi) \frac{\partial u(\mathrm{x})}{\partial \mathbf{n}}\right] d C_{\rho} \\
=\sum_{k=1}^{n} \int_{S_{k}} G_{k}^{*}\left(x_{1}, \cdots, x_{k-1}, 0, x_{k+1}, \cdots, x_{m} ; \xi\right) \tau_{k}\left(\tilde{x}_{k}\right) d S_{k} \\
+\int_{S} \mathbf{x}^{(2 \alpha)} \frac{\partial G(\mathbf{x} ; \xi)}{\partial \mathbf{n}} \varphi(S) d S,
\end{gathered}
$$

where

$$
\begin{gathered}
\mathrm{x}^{(2 \alpha)}:=x_{1}^{2 \alpha_{1}} \cdots x_{n}^{2 \alpha_{n}}, \quad \tilde{x}_{k}^{(2 \alpha)}:=x_{1}^{2 \alpha_{1}} \cdots x_{k-1}^{2 \alpha_{k-1}} x_{k+1}^{2 \alpha_{k+1}} \cdots x_{n}^{2 \alpha_{n}} \\
G_{k}^{*}\left(x_{1}, \cdots, x_{k-1}, 0, x_{k+1}, \cdots, x_{m} ; \xi\right)=\left.\tilde{x}_{k}^{(2 \alpha)} \cdot\left(x_{k}^{2 \alpha_{k}} \frac{\partial G(\mathrm{x} ; \xi)}{\partial x_{k}}\right)\right|_{x_{k}=0} \\
\left(x_{1}, \cdots, x_{k-1}, 0, x_{k+1}, \cdots, x_{m}\right) \in S_{k}
\end{gathered}
$$

First, we consider an integral

$$
\int_{C_{\rho}} \mathbf{x}^{(2 \alpha)} u(\mathrm{x}) \frac{\partial G(\mathrm{x} ; \xi)}{\partial \mathbf{n}} d C_{\rho} .
$$

Taking (24) into account we rewrite it as follows

$$
\begin{gathered}
\int_{C_{\rho}} \mathbf{x}^{(2 \alpha)} u \frac{\partial G}{\partial \mathbf{n}} d C_{\rho} \\
\equiv \int_{C_{\rho}} \mathbf{x}^{(2 \alpha)} u \frac{\partial q_{n}(\mathbf{x} ; \xi)}{\partial \mathbf{n}} d C_{\rho}+\int_{C_{\rho}} \mathbf{x}^{(2 \alpha)} u \frac{\partial q_{n}^{*}(\mathbf{x} ; \xi)}{\partial \mathbf{n}} d C_{\rho}=I_{1}+I_{2} .
\end{gathered}
$$

Using the formula of differentiation

$$
\begin{aligned}
& \frac{\partial}{\partial z_{j}} F_{A}^{(n)}\left(a, b_{1}, \cdots, b_{n} ; c_{1}, \cdots, c_{n} ; z_{1}, \cdots, z_{n}\right) \\
& =\frac{a b_{j}}{c_{j}} F_{A}^{(n)}\left(a+1, b_{1}, \cdots, b_{j-1}, b_{j}+1,\right. \\
& \left.b_{j+1}, \cdots, b_{n} ; c_{1}, \cdots, c_{j-1}, c_{j}+1, c_{j+1}, \cdots, c_{n} ; z_{1}, \cdots, z_{n}\right)
\end{aligned}
$$


and the following adjacent relation

$$
\begin{gathered}
\sum_{j=1}^{n} \frac{b_{j}}{c_{j}} z_{j} F_{A}^{(n)}\left(a+1, b_{1}, \cdots, b_{j-1}, b_{j}+1, b_{j+1}, \cdots, b_{n} ;\right. \\
\left.c_{1}, \cdots, c_{j-1}, c_{j}+1, c_{j+1}, \cdots, c_{n} ; z_{1}, \cdots, z_{n}\right) \\
=F_{A}^{(n)}\left(a+1, b_{1}, \cdots, b_{n} ; c_{1}, \cdots, c_{n} ; z_{1}, \cdots, z_{n}\right) \\
\quad-F_{A}^{(n)}\left(a, b_{1}, \cdots, b_{n} ; c_{1}, \cdots, c_{n} ; z_{1}, \cdots, z_{n}\right),
\end{gathered}
$$

we calculate

$$
\frac{\partial q_{n}(\mathrm{x} ; \xi)}{\partial \mathbf{n}}=\sum_{i=1}^{m} \frac{\partial q_{n}(\mathrm{x} ; \xi)}{\partial x_{i}} \cdot \cos \left(\mathbf{n}, x_{i}\right) .
$$

Below we get detailed evaluations for $\frac{\partial q_{n}(\mathrm{x} ; \xi)}{\partial x_{i}}$, when $1 \leq i \leq n$. Indeed, using the formula of differentiation (26), we get

$$
\begin{gathered}
\frac{\partial q_{n}(x, \xi)}{\partial x_{k}}=\left(1-2 \alpha_{k}\right) \gamma_{n} x_{k}^{-2 \alpha_{k}} \xi_{k}^{1-2 \alpha_{k}} \\
\times \prod_{i=1, i \neq k}^{n}\left[\left(x_{i} \xi_{i}\right)^{1-2 \alpha_{i}}\right] r^{-2 \bar{\alpha}_{n}} F_{A}^{(n)}\left[\begin{array}{c}
\bar{\alpha}_{n}, 1-\alpha_{1}, \cdots, 1-\alpha_{n} ; \\
2-2 \alpha_{1}, \cdots, 2-2 \alpha_{n} ;
\end{array}\right] \\
-2 \bar{\alpha}_{n} \gamma_{n}\left(x_{k}-\xi_{k}\right) \prod_{i=1}^{n}\left[\left(x_{i} \xi_{i}\right)^{1-2 \alpha_{i}}\right] r^{-2 \bar{\alpha}_{n}-2} F_{A}^{(n)}\left[\begin{array}{c}
\bar{\alpha}_{n}, 1-\alpha_{1}, \cdots, 1-\alpha_{n} ; \\
2-2 \alpha_{1}, \cdots, 2-2 \alpha_{n} ;
\end{array}\right] \\
-2 \bar{\alpha}_{n} \gamma_{n} \xi_{k} \prod_{i=1}^{n}\left[\left(x_{i} \xi_{i}\right)^{1-2 \alpha_{i}}\right] r^{-2 \bar{\alpha}_{n}-2} \\
\times F_{A}^{(n)}\left[\begin{array}{c}
\bar{\alpha}_{n}+1,1-\alpha_{1}, \cdots, 1-\alpha_{k-1}, 2-\alpha_{k}, 1-\alpha_{k+1}, \cdots 1-\alpha_{n} ; \\
2-2 \alpha_{1}, \cdots, 2-2 \alpha_{k-1}, 3-2 \alpha_{k}, 2-2 \alpha_{k+1}, \cdots 2-2 \alpha_{n} ;
\end{array}\right] \\
-2 \bar{\alpha}_{n} \gamma_{n}\left(x_{k}-\xi_{k}\right) \prod_{i=1}^{n}\left[\left(x_{i} \xi_{i}\right)^{1-2 \alpha_{i}}\right] r^{-2 \bar{\alpha}_{n}-2} \sum_{i=1}^{n} \frac{1-\alpha_{i}}{2-2 \alpha_{i}} \sigma_{i} \\
\times F_{A}^{(n)}\left[\begin{array}{c}
\bar{\alpha}_{n}+1,1-\alpha_{1}, \cdots, 1-\alpha_{i-1}, 2-\alpha_{i}, 1-\alpha_{i+1}, \cdots 1-\alpha_{n} ; \\
2-2 \alpha_{1}, \cdots, 2-2 \alpha_{i-1}, 3-2 \alpha_{i}, 2-2 \alpha_{i+1}, \cdots 2-2 \alpha_{n} ;
\end{array}\right] .
\end{gathered}
$$


Considering adjacent relation (27) we obtain

$$
\begin{aligned}
& \frac{\partial q_{n}(x, \xi)}{\partial x_{k}}=\left(1-2 \alpha_{k}\right) \gamma_{n} x_{k}^{-2 \alpha_{k}} \xi_{k}^{1-2 \alpha_{k}} \\
& \times \prod_{i=1, i \neq k}^{n}\left[\left(x_{i} \xi_{i}\right)^{1-2 \alpha_{i}}\right] r^{-2 \tilde{\alpha}_{n}} F_{A}^{(n)}\left[\begin{array}{c}
\bar{\alpha}_{n}, 1-\alpha_{1}, \cdots, 1-\alpha_{n} ; \\
2-2 \alpha_{1}, \cdots, 2-2 \alpha_{n} ;
\end{array}\right] \\
& -2 \bar{\alpha}_{n} \gamma_{n} \xi_{k} \prod_{i=1}^{n}\left[\left(x_{i} \xi_{i}\right)^{1-2 \alpha_{i}}\right] r^{-2 \bar{\alpha}_{n}-2} \\
& \times F_{A}^{(n)}\left[\begin{array}{c}
\bar{\alpha}_{n}+1,1-\alpha_{1}, \cdots, 1-\alpha_{k-1}, 2-\alpha_{k}, 1-\alpha_{k+1}, \cdots 1-\alpha_{n} \\
2-2 \alpha_{1}, \cdots, 2-2 \alpha_{k-1}, 3-2 \alpha_{k}, 2-2 \alpha_{k+1}, \cdots 2-2 \alpha_{n}
\end{array}\right] \\
& +2 \bar{\alpha}_{n} \gamma_{n}\left(\xi_{k}-x_{k}\right) \prod_{i=1}^{n}\left[\left(x_{i} \xi_{i}\right)^{1-2 \alpha_{i}}\right] r^{-2 \bar{\alpha}_{n}-2} \\
& \times F_{A}^{(n)}\left[\begin{array}{c}
\bar{\alpha}_{n}+1,1-\alpha_{1}, \cdots, 1-\alpha_{n} ; \\
2-2 \alpha_{1}, \cdots, 2-2 \alpha_{n} ;
\end{array}\right] .
\end{aligned}
$$

Similarly we calculate $\frac{\partial q_{n}(\mathrm{x} ; \xi)}{\partial x_{i}}$, when $n+1 \leq i \leq m$ :

$$
\begin{gathered}
\frac{\partial q_{n}(x, \xi)}{\partial x_{k}}=2 \bar{\alpha}_{n} \gamma_{n}\left(\xi_{k}-x_{k}\right) \prod_{i=1}^{n}\left[\left(x_{i} \xi_{i}\right)^{1-2 \alpha_{i}}\right] \\
\times r^{-2 \bar{\alpha}_{n}-2} F_{A}^{(n)}\left[\begin{array}{c}
\bar{\alpha}_{n}+1,1-\alpha_{1}, \cdots, 1-\alpha_{n} ; \\
2-2 \alpha_{1}, \cdots, 2-2 \alpha_{n} ;
\end{array}\right] .
\end{gathered}
$$

Taking (28), (29) and (30) into account we calculate

$$
\begin{gathered}
\frac{\partial q_{n}(x, \xi)}{\partial \mathbf{n}}=-\bar{\alpha}_{n} \gamma_{n} \prod_{i=1}^{n}\left[\left(x_{i} \xi_{i}\right)^{1-2 \alpha_{i}}\right] r^{-2 \bar{\alpha}_{n}} \\
\times F_{A}^{(n)}\left[\begin{array}{c}
\bar{\alpha}_{n}+1,1-\alpha_{1}, \cdots, 1-\alpha_{n} ; \\
2-2 \alpha_{1}, \cdots, 2-2 \alpha_{n} ;
\end{array}\right] \frac{\partial}{\partial \mathbf{n}}\left[\ln r^{2}\right] \\
-2 \bar{\alpha}_{n} \gamma_{n} \prod_{i=1}^{n}\left[\left(x_{i} \xi_{i}\right)^{1-2 \alpha_{i}}\right] r^{-2 \bar{\alpha}_{n}-2} \cos \left(\mathbf{n} ; x_{i}\right) \\
\times \sum_{i=1}^{n} \xi_{i} F_{A}^{(n)}\left[\begin{array}{c}
\bar{\alpha}_{n}+1,1-\alpha_{1}, \cdots, 1-\alpha_{i-1}, 2-\alpha_{i}, 1-\alpha_{i+1}, \cdots 1-\alpha_{n} ; \\
2-2 \alpha_{1}, \cdots, 2-2 \alpha_{i-1}, 3-2 \alpha_{i}, 2-2 \alpha_{i+1}, \cdots 2-2 \alpha_{n} ;
\end{array}\right]
\end{gathered}
$$




$$
\begin{gathered}
+\gamma_{n} \prod_{i=1}^{n}\left[\left(x_{i} \xi_{i}\right)^{1-2 \alpha_{i}}\right] r^{-2 \bar{\alpha}_{n}} \\
\times F_{A}^{(n)}\left[\begin{array}{c}
\bar{\alpha}_{n}, 1-\alpha_{1}, \cdots, 1-\alpha_{n} ; \\
2-2 \alpha_{1}, \cdots, 2-2 \alpha_{n} ;
\end{array}\right] \cdot \sum_{i=1}^{n} \frac{1-2 \alpha_{i}}{x_{i}} \cos \left(\mathbf{n} ; x_{i}\right) .
\end{gathered}
$$

Now consider the integral

$$
\int_{C_{\rho}} \mathbf{x}^{(2 \alpha)} u \frac{\partial q_{n}(\mathrm{x} ; \xi)}{\partial \mathbf{n}} d C_{\rho}=I_{11}+I_{12}+I_{13},
$$

where

$$
\begin{gathered}
I_{11}=-\bar{\alpha}_{n} \gamma_{n} \prod_{i=1}^{n}\left[\xi_{i}^{1-2 \alpha_{i}}\right] \int_{C_{\rho}} u \cdot \prod_{i=1}^{n}\left[x_{i}\right] r^{-2 \bar{\alpha}_{n}} \\
\times F_{A}^{(n)}\left[\begin{array}{c}
\left.\bar{\alpha}_{n}+1,1-\alpha_{1}, \cdots, 1-\alpha_{n} ; \sigma\right] \\
2-2 \alpha_{1}, \cdots, 2-2 \alpha_{n} ;
\end{array}\right] \frac{\partial}{\partial \mathbf{n}}\left[\ln r^{2}\right] d C_{\rho}, \\
I_{12}=-2 \bar{\alpha}_{n} \gamma_{n} \prod_{i=1}^{n}\left[\xi_{i}^{1-2 \alpha_{i}}\right] \int_{C_{\rho}} u \cdot \prod_{i=1}^{n}\left[x_{i}\right] r^{-2 \bar{\alpha}_{n}-2} \sum_{i=1}^{n} \xi_{i} \cdot \cos \left(\mathbf{n} ; x_{i}\right) \\
\times F_{A}^{(n)}\left[\begin{array}{c}
\bar{\alpha}_{n}+1,1-\alpha_{1}, \cdots, 1-\alpha_{i-1}, 2-\alpha_{i}, 1-\alpha_{i+1}, \cdots 1-\alpha_{n} ; \\
2-2 \alpha_{1}, \cdots, 2-2 \alpha_{i-1}, 3-2 \alpha_{i}, 2-2 \alpha_{i+1}, \cdots 2-2 \alpha_{n} ;
\end{array}\right] d C_{\rho}, \\
I_{13}=\gamma_{n} \prod_{i=1}^{n}\left[\xi_{i}^{1-2 \alpha_{i}}\right] \int_{C_{\rho}} u \cdot \prod_{i=1}^{n}\left[x_{i}\right] r^{-2 \bar{\alpha}_{n}} \\
\times F_{A}^{(n)}\left[\begin{array}{c}
\bar{\alpha}_{n}, 1-\alpha_{1}, \cdots, 1-\alpha_{n} ; \\
2-2 \alpha_{1}, \cdots, 2-2 \alpha_{n} ;
\end{array}\right] \sum_{i=1}^{n} \frac{1-2 \alpha_{i}}{x_{i}} \cos \left(\mathbf{n} ; x_{i}\right) d C_{\rho} .
\end{gathered}
$$

We use the following generalization spherical system of coordinates:

$$
\begin{gathered}
x_{1}=\xi_{1}+\rho \cos \varphi_{1}, \\
x_{2}=\xi_{2}+\rho \sin \varphi_{1} \cos \varphi_{2}, \\
x_{3}=\xi_{3}+\rho \sin \varphi_{1} \sin \varphi_{2} \cos \varphi_{3}, \\
\vdots \\
x_{m-1}=\xi_{m-1}+\rho \sin \varphi_{1} \sin \varphi_{2} \cdots \sin \varphi_{m-2} \cos \varphi_{m-1}, \\
x_{m}=\xi_{m}+\rho \sin \varphi_{1} \sin \varphi_{2} \cdots \sin \varphi_{m-2} \sin \varphi_{m-1},
\end{gathered}
$$




$$
\left[\rho \geq 0,0 \leq \varphi_{1} \leq \pi, \cdots, 0 \leq \varphi_{m-2} \leq \pi, 0 \leq \varphi_{m-1} \leq 2 \pi\right] .
$$

Then we have

$$
\begin{gathered}
I_{11}=2 \bar{\alpha}_{n} \gamma_{n} \prod_{i=1}^{n}\left[\xi_{i}^{1-2 \alpha_{i}}\right] \rho^{2 \alpha_{1}+\cdots+2 \alpha_{n}-2 n} \int_{0}^{2 \pi} d \varphi_{m-1} \\
\int_{0}^{\pi} \sin \varphi_{m-2} d \varphi_{m-2} \cdots \int_{0}^{\pi} \tilde{u} v F_{A}^{(n)}(\tilde{\sigma}) \sin ^{m-2} \varphi_{1} d \varphi_{1},
\end{gathered}
$$

where

$$
\begin{aligned}
& \tilde{u}:=u\left(\xi_{1}+\rho \cos \varphi_{1}, \xi_{2}+\rho \sin \varphi_{1} \cos \varphi_{2}, \xi_{3}+\rho \sin \varphi_{1} \sin \varphi_{2} \cos \varphi_{3}, \cdots,\right. \\
& \xi_{m-1}+\rho \sin \varphi_{1} \sin \varphi_{2} \cdots \sin \varphi_{m-2} \cos \varphi_{m-1}, \\
& \xi_{m}\left.+\rho \sin \varphi_{1} \sin \varphi_{2} \cdots \sin \varphi_{m-2} \sin \varphi_{m-1}\right), \\
& v:=\left(\xi_{1}+\rho \cos \varphi_{1}\right)\left(\xi_{2}+\rho \sin \varphi_{1} \cos \varphi_{2}\right)\left(\xi_{3}+\rho \sin \varphi_{1} \sin \varphi_{2} \cos \varphi_{3}\right) \cdots \\
& \cdots\left(\xi_{m-1}+\rho \sin \varphi_{1} \sin \varphi_{2} \cdots \sin \varphi_{m-2} \cos \varphi_{m-1}\right) \\
& \times\left(\xi_{m}+\rho \sin \varphi_{1} \sin \varphi_{2} \cdots \sin \varphi_{m-2} \sin \varphi_{m-1}\right), \\
& F_{A}^{(n)}(\tilde{\sigma}):=F_{A}^{(n)}\left(\bar{\alpha}_{n}+1,1-\alpha_{1}, \cdots, 1-\alpha_{n} ; 2-2 \alpha_{1}, \cdots, 2-2 \alpha_{n} ; \tilde{\sigma}\right), \\
& \tilde{\sigma}:=\left(\sigma_{1 \rho}, \cdots, \sigma_{n \rho}\right) ; r_{k}^{2}=\left(x_{k}+\xi_{k}\right)^{2}+\sum_{i=1, i \neq k}^{m}\left(x_{i}-\xi_{i}\right)^{2}, \sigma_{k \rho}=1-\frac{r_{k \rho}^{2}}{\rho^{2}} .
\end{aligned}
$$

First we evaluate $F_{A}^{(n)}(\tilde{\sigma})$. For this aim we use decomposition formula (13) and then auto-transformation formula (7):

$$
\begin{gathered}
F_{A}^{(n)}(\tilde{\sigma})=\sum_{\substack{m_{i, j}=0 \\
(2 \leq i \leq j \leq n)}}^{\infty} \frac{\left(\bar{\alpha}_{n}+1\right)_{A(n, n)}}{m_{i j} !} \prod_{k=1}^{n}\left[\frac{\left(1-\alpha_{k}\right)_{B(k, n)}}{\left(2-2 \alpha_{k}\right)_{B(k, n)}}\left(\frac{r_{k \rho}^{2}}{\rho^{2}}\right)^{\alpha_{k}-1-B(k, n)}\right. \\
\left.\times\left(1-\frac{r_{k \rho}^{2}}{\rho^{2}}\right)^{B(k, n)}\right] \prod_{k=1}^{n}\left[F \left(1-2 \alpha_{k}-\bar{\alpha}_{n}+B(k, n)-A(k, n),\right.\right. \\
\left.\left.1-\alpha_{k}+B(k, n) ; 2-2 \alpha_{k}+B(k, n) ; \frac{\sigma_{k \rho}}{\sigma_{k \rho}-1}\right)\right],
\end{gathered}
$$

where $A(k, n)$ and $B(k, n)$ are an expressions defined in (14). 
After the elementary evaluations we find

$$
F_{A}^{(n)}(\tilde{\sigma})=\rho^{2 n-2 \alpha_{1}-\cdots-2 \alpha_{n}} \prod_{k=1}^{n}\left[r_{k \rho}^{2 \alpha_{k}-2}\right] \cdot \aleph
$$

where

$$
\begin{aligned}
\aleph:=\sum_{\substack{m_{i, j}=0 \\
(2 \leq i \leq j \leq n)}}^{\infty} \frac{\left(\bar{\alpha}_{n}+1\right)_{A(n, n)}}{m_{i j} !} \prod_{k=1}^{n}\left[\frac{\left(1-\alpha_{k}\right)_{B(k, n)}}{\left(2-2 \alpha_{k}\right)_{B(k, n)}}\left(\frac{\rho^{2}}{r_{k \rho}^{2}}-1\right)^{B(k, n)}\right] \\
\times \prod_{k=1}^{n}\left[F \left(1-2 \alpha_{k}-\bar{\alpha}_{n}+B(k, n)-A(k, n),\right.\right. \\
\left.\left.1-\alpha_{k}+B(k, n) ; 2-2 \alpha_{k}+B(k, n) ; 1-\frac{\rho^{2}}{r_{k \rho}^{2}}\right)\right] .
\end{aligned}
$$

It is easy to see that when $\rho \rightarrow 0$ the function $\aleph$ becomes an expression that does not depend on $\mathrm{x}$ and $\xi$. Indeed, taking into account the equality (15), we have

$$
\begin{aligned}
& \lim _{\rho \rightarrow 0} \aleph=\sum_{\substack{m_{i, j}=0 \\
(2 \leq i \leq j \leq n)}}^{\infty} \frac{\left(\bar{\alpha}_{n}+1\right)_{A(n, n)}}{m_{i j} !} \prod_{k=1}^{n}\left[\frac{\left(1-\alpha_{k}\right)_{B(k, n)}}{\left(2-2 \alpha_{k}\right)_{B(k, n)}}\right] \prod_{k=1}^{n}\left[F \left(1-2 \alpha_{k}\right.\right. \\
& \left.\left.-\bar{\alpha}_{n}+B(k, n)-A(k, n), 1-\alpha_{k}+B(k, n) ; 2-2 \alpha_{k}+B(k, n) ; 1\right)\right] .
\end{aligned}
$$

Applying now the summation formula (6) to each hypergeometric function $F(a, b ; c ; 1)$ in the sum $(32)$, we get

$$
\begin{gathered}
\lim _{\rho \rightarrow 0} \aleph=\frac{1}{\Gamma\left(\bar{\alpha}_{n}+1\right)} \sum_{\substack{m_{i, j}=0 \\
(2 \leq i \leq j \leq n)}}^{\infty} \frac{\Gamma\left(\bar{\alpha}_{n}+1+A(n, n)\right)}{m_{i j} !} \\
\times \prod_{k=1}^{n}\left[\frac{\Gamma\left(1-\alpha_{k}+B(k, n)\right) \Gamma\left(2-2 \alpha_{k}\right) \Gamma\left(\bar{\alpha}_{n}+\alpha_{k}+A(k, n)-B(k, n)\right)}{\Gamma\left(\bar{\alpha}_{n}+1+A(k, n)\right) \Gamma^{2}\left(1-\alpha_{k}\right)}\right] .
\end{gathered}
$$

Taking into account the identity

$$
\sum_{\substack{m_{i, j}=0 \\(2 \leq i \leq j \leq n)}}^{\infty} \frac{\left(\bar{\alpha}_{n}+1\right)_{A(n, n)}}{m_{i j} !} \prod_{k=1}^{n}\left[\frac{\left(\bar{\alpha}_{n}+\alpha_{k}\right)_{A(k, n)-B(k, n)}\left(1-\alpha_{k}\right)_{B(k, n)}}{\left(\bar{\alpha}_{n}+1\right)_{B(k, n)}}\right]
$$




$$
\begin{gathered}
=\Gamma\left(\frac{m}{2}\right) \frac{\Gamma^{n-1}\left(\bar{\alpha}_{n}+1\right)}{\prod_{k=1}^{n} \Gamma\left(\bar{\alpha}_{n}+\alpha_{k}\right)}, \\
{\left[m \geq 2,0<n \leq m ; 0<2 \alpha_{k}<1, \quad k=\overline{1, n}\right]}
\end{gathered}
$$

we obtain

$$
\lim _{\rho \rightarrow 0} \aleph=\frac{\Gamma(m / 2)}{\Gamma\left(\bar{\alpha}_{n}+1\right)} \prod_{i=1}^{n} \frac{\Gamma\left(2-2 \alpha_{k}\right)}{\Gamma\left(1-\alpha_{k}\right)},
$$

where $A(k, n), B(k, n)$ and $\bar{\alpha}_{n}$ are expressions defined in (14) and (17).

Using the properties (4) of gamma-function $\Gamma(z)$, property (5) of the Pochhammer symbol and summation formula (6) for hypergeometric function $F(a, b ; c ; z)$, the formula (33) is proved by the method mathematical induction.

Now we consider an integral

$$
L_{m}=\int_{0}^{2 \pi} d \varphi_{m-1} \int_{0}^{\pi} \sin \varphi_{m-2} d \varphi_{m-2} \int_{0}^{\pi} \sin ^{2} \varphi_{m-3} d \varphi_{m-3} \cdots \int_{0}^{\pi} \sin ^{m-2} \varphi_{1} d \varphi_{1} .
$$

with elementary transformations it is not difficult to establish that

$$
L_{2 m}=\frac{2 \pi^{m}}{(m-1) !}, L_{2 m+1}=\frac{2^{m+1} \pi^{m}}{(2 m-1) ! !}, \quad m=1,2,3, \cdots
$$

If we take into account (31), (34), (35) and (18), then we will have

$$
\lim _{\rho \rightarrow 0} I_{11}=u(\xi)
$$

By similar evaluations one can get that

$$
\lim _{\rho \rightarrow 0} I_{12}=\lim _{\rho \rightarrow 0} I_{13}=\lim _{\rho \rightarrow 0} I_{2}=0 .
$$

If we consider an integral

$$
\int_{C_{\rho}} \mathrm{x}^{(2 \alpha)} G(\mathrm{x} ; \xi) \frac{\partial u(\mathrm{x})}{\partial \mathbf{n}} d C_{\rho}
$$

using above given algorithm for evaluations (in this case calculations will be more simple), we can prove that

$$
\lim _{\rho \rightarrow 0} \int_{C_{\rho}} \mathrm{x}^{(2 \alpha)} G(\mathrm{x} ; \xi) \frac{\partial u(\mathrm{x})}{\partial \mathbf{n}} d C_{\rho}=0 .
$$


Now from (25) we can write the solution of the Dirichlet problem as follows:

$$
\begin{aligned}
u(\xi)=\sum_{k=1}^{n} \int_{S_{k}} G_{k}^{*} & \left(x_{1}, \cdots, x_{k-1}, 0, x_{k+1}, \cdots, x_{m} ; \xi\right) \tau_{k}\left(\tilde{x}_{k}\right) d S_{k} \\
& +\int_{S} x^{(2 \alpha)} \frac{\partial G(\mathrm{x} ; \xi)}{\partial \mathbf{n}} \varphi(\mathrm{x}) d S .
\end{aligned}
$$

The particular values of Green's function are given by

$$
\begin{gathered}
G_{k}^{*}\left(x_{1}, \cdots, x_{k-1}, 0, x_{k+1}, \cdots, x_{m} ; \xi\right) \\
=\left(1-2 \alpha_{k}\right) \gamma_{n} \prod_{i=1}^{n}\left[\xi_{i}^{1-2 \alpha_{i}}\right] \cdot \prod_{i=1, i \neq k}^{n}\left[x_{i}\right] \\
\times\left\{\frac{F_{A}^{(n-1)}\left[\begin{array}{c}
\bar{\alpha}_{n}, 1-\alpha_{1}, \cdots, 1-\alpha_{k-1}, 1-\alpha_{k+1}, \cdots, 1-\alpha_{n} ; \\
2-2 \alpha_{1}, \cdots, 2-2 \alpha_{k-1}, 2-2 \alpha_{k+1}, \cdots, 2-2 \alpha_{n} ;
\end{array}\right]}{\left[\xi_{k}^{2}+\sum_{i=1, i \neq k}^{m}\left(\xi_{i}-x_{i}\right)^{2}\right]^{\bar{\alpha}_{n}}}\right. \\
\left.-\frac{F_{A}^{(n-1)}\left[\begin{array}{c}
\bar{\alpha}_{n}, 1-\alpha_{1}, \cdots, 1-\alpha_{k-1}, 1-\alpha_{k+1}, \cdots, 1-\alpha_{n} ; \\
2-2 \alpha_{1}, \cdots, 2-2 \alpha_{k-1}, 2-2 \alpha_{k+1}, \cdots, 2-2 \alpha_{n} ;
\end{array}\right]}{\left[\sum_{i=1, i \neq k}^{m}\left(a-\frac{x_{i} \xi_{i}}{a}\right)^{2}+\frac{1}{a^{2}} \sum_{i=1, i \neq k}^{m} \sum_{j=1, j \neq i}^{m} x_{i}^{2} \xi_{j}^{2}-(m-2) a^{2}\right]^{\bar{\alpha}_{n}}}\right\},
\end{gathered}
$$

where

$$
\begin{gathered}
\sigma_{0}:=\left(\sigma_{1}^{0}, \cdots, \sigma_{k-1}^{0}, \sigma_{k+1}^{0}, \cdots, \sigma_{n}^{0}\right), \bar{\sigma}_{0}:=\left(\bar{\sigma}_{1}^{0}, \cdots, \bar{\sigma}_{k-1}^{0}, \bar{\sigma}_{k+1}^{0}, \cdots, \bar{\sigma}_{n}^{0}\right), \\
\bar{\sigma}_{s}^{0}=-\frac{a^{2}}{R_{0}^{2}} \frac{4 x_{s} \xi_{s}}{\sum_{i=1, i \neq k}^{m}\left(a-\frac{x_{i} \xi_{i}}{a}\right)^{2}+\frac{1}{a^{2}} \sum_{i=1, i \neq k}^{m} \sum_{j=1, j \neq i}^{m} x_{i}^{2} \xi_{j}^{2}-(m-2) a^{2}}, \\
\sigma_{s}^{0}=-\frac{4 x_{s} \xi_{s}}{\xi_{k}^{2}+\sum_{i=1, i \neq k}^{m}\left(\xi_{i}-x_{i}\right)^{2}}, s=\overline{1, n}, s \neq k .
\end{gathered}
$$

Constant $\gamma_{n}$ has the form (18).

Hence, the main result of the paper is formulated as the following theorem:

Theorem 4.2. If $\tau_{k}\left(\tilde{x}_{k}\right) \in C^{2}\left(S_{k}\right)$ and $\varphi(\mathrm{x}) \in C^{2}(S)$ are given functions fulfilling the matching conditions (21), then the Dirichlet problem has unique solution represented by formula (36). 


\section{Bibliography}

[1] C. Agostinelli, Integrazione dell'equazione differenziale $u_{x x}+u_{y y}+u_{z z}+x^{-1} u_{x}=$ $f$ e problema analogo a quello di Dirichlet per un campo emisferico, Atti della Accademia Nazionale dei Lincei 6(26) (1937), 7-8 (In Italian).

[2] A. Altin, Solutions of type $r^{m}$ for a class of singular equations, Intern. Jour. of Math. Sc. 5(3) (1982), 613-619.

[3] L. Bers, Mathematical Aspects of Subsonic and Transonic Gas Dynamics, New York, London, 1958.

[4] A. V. Bitsadze, Some Classes of Partial Differential Equations, Nauka, Moscow, 1981 (In Russian).

[5] J. L. Burchnall and T. W. Chaundy, Expansions of Appell's double hypergeometric functions, Quart. J. Math. Oxford 11 (1940), 249-270.

[6] J. L. Burchnall and T. W. Chaundy, Expansions of Appell's double hypergeometric functions II, Quart. J. Math. Oxford 12 (1941), 112-128.

[7] A. Erdelyi, W. Magnus, F. Oberhettinger and F. G. Tricomi, Higher Transcendental Functions, Vol. I, McGraw-Hill Book Company, New York, Toronto and London, 1953.

[8] T. G. Ergashev, Fundamental solutions for a class of multidimensional elliptic equations with several singular coefficients, preprint (2018), http: //arxiv.org/abs/1805.03826.

[9] T. G. Ergashev, Third double-layer potential for a generalized bi-axially symmetric Helmholtz equation, Ufa Mathematical Journal 10(4) (2018), 111-122.

[10] T. G. Ergashev, The fourth double-layer potential for a generalized bi-axially symmetric Helmholtz equation, Tomsk State University Journal of Mathematics and Mechanics (Vestnik Tomskogo gosudarstvennogo universiteta. Matematika i mekhanika) 50 (2017), 45-56, doi:10.17223/19988621/50/4 (In Russian).

[11] F. I. Frankl, On the problems of Chapligin for mixed sub- and supersonic flows, Bull. Sci. USSR. Ser. Math. 9 (1945), 121-123.

[12] A. J. Fryant, Growth and complete sequences of generalized bi-axially symmetric potentials, Jour. of Diff. Eq. 31(2) (1979), 155-164.

[13] R. Gilbert, On the location of singularities of a class of elliptic partial differential equations in four variables, Canadian Journal Mathematics 17 (1965), 676-686.

[14] R. Gilbert, Function Theoretic Methods in Partial Differential Equations, Academic Press, New York-London, 1969.

[15] A. Hasanov, Fundamental solutions of generalized bi-axially symmetric Helmholtz equation, Complex Variables and Elliptic Equations 52(8) (2007), 673-683, doi:10.1080/17476930701300375. 
[16] A. Hasanov and E. T. Karimov, Fundamental solutions for a class of threedimensional elliptic equations with singular coefficients, Appl. Math. Lett. 22 (2009), 1828-1832, doi:10.10/j.aml.2009.07.006.

[17] A. Hasanov and H. M. Srivastava, Some decomposition formulas associated with the Lauricella function $F_{A}^{(r)}$ and other multiple hypergeometric functions, Appl. Math. Lett. 19(2) (2006), 113-121, doi:10.1016/j.aml.2005.03.009.

[18] A. Hasanov and H. M. Srivastava, Decomposition Formulas Associated with the Lauricella Multivariable Hypergeometric Functions, Comput. and Math. with Appl. 53(7) (2007), 1119-1128, doi:10.1016/j.camwa.2006.07.007.

[19] P. Henrici, On the domain of regularity of generalized axially symmetric potentials, Proc. Amer. Math. Soc. 8 (1957), 29-31.

[20] E. T. Karimov, On a boundary problem with Neumann's condition for 3D singular elliptic equations, Appl. Math. Lett. 23 (2010), 517-522, doi:10.1016/j.aml.2010.01.002.

[21] E. T. Karimov and J. J. Nieto, The Dirichlet problem for a 3D elliptic equation with two singular coefficients, Comput. Math. Appl. 62 (2011), 214-224, doi:10.1016/j.camwa.2011.04.068.

[22] P. Kumar, Approximation of growth numbers generalized bi-axially symmetric potentials, Fasciculi Mathematics 55 (2005), 51-60.

[23] G. Lauricella, Sulle funzione ipergeometriche a più variabili, Rend.Circ. Mat. Palermo 7 (1893), 111-158 (In Italian).

[24] P. A. McCoy, Polynomial approximation and growth of generalized axisymmetric potentials, Canadian Journal Mathematics 31(1) (1979), 49-59.

[25] I. T. Nazipov, Solution of the spatial Tricomi problem for a singular mixed-type equation by the method of integral equations, Russian Mathematics 55(3) (2011), 61-76.

[26] J. J. Nieto and E. T. Karimov, On an anologue of the Holmgreen's problem for 3D singular elliptic equation, Asian-European Jour. of Math. 5(2) (2012), 1-18, doi:10.1142/S1793557112500210.

[27] M. N. Olevskii, Solution of the Dirichlet problem for the equation for a hemispherical region, Dokl.Akad.Nauk SSSR 64 (1949), 767-770.

[28] M. Rassias, Lecture Notes on Mixed Type Partial Differential Equations, World Scientific, 1990.

[29] M. S. Salakhitdinov and A. Hasanov, The Dirichlet problem for generalized biaxially symmetric Helmholtz equation, Eurasian Mathematical Journal 3(4) (2008), 99-110. 
[30] M. S. Salakhitdinov and A. Hasanov, A solution of the Neumann-Dirichlet boundary value problem for generalized bi-axially symmetric Helmholtz equation, Complex Variables and Elliptic Equations 53(4) (2008), 355-364, doi:10.1080/17476930701769041.

[31] M. S. Salakhitdinov and A. Hasanov, The boundary problem $N D_{1}$ for generalized axially symmetric Helmholtz equation, Reports of International Academy of Sciences of Adygey 13(1) (2011), 109-116.

[32] M. S. Salakhitdinov and E. T. Karimov, Spatial boundary problem with the DirichletNeumann condition for a singular elliptic equation, Appl. Math. and Comput. 219 (2012), 3469-3476, doi:10.1016/j.amc,2012.09.013.

[33] M. S. Salakhitdinov and M. Mirsaburov, Nonlocal Boundary Value Problems for Equations of Mixed Type with Singular Coefficients, Universitet, Tashkent, 2005 (In Russian).

[34] M. M. Smirnov, Degenerating Elliptic and Hyperbolic Equations, Nauka, Moscow, 1966 (In Russian).

[35] H. M. Srivastava, A. Hasanov and J. Choi, Double-layer potentials for a generalized bi-axially symmetric Helmholtz equation, Sohag Journal of Mathematics 2(1)(2015), 1-10, doi: 10.12785/sjm/020101.

[36] R. J. Weinacht, Some properties of generalized axially symmetric Helmholtz potentials, SIAM Jour. Math. Anal. 5 (1974), 147-152.

Received December 29, 2018; revised May 4, 2019; accepted May 7, 2019.

\section{Author information}

Tuhtasin G. Ergashev, V. I. Romanovskiy Institute of Mathematics, Uzbekistan Academy of Sciences, 100170, Tashkent, Uzbekistan.

E-mail: ergashev.tukhtasin@gmail.com 